\title{
Popova, A.A., Anodnoe Povedenie Perekhodnykh Metallov $v$ Vodnykh $i$ Organicheskikh Sredakh (Anodic Behavior of Transition Metals in Aqueous and Organic Media),
}

\author{
Rostov-on-Don: Izd. SKNTs VSh, 2006, 184 pp.
}

DOI: $10.1134 / \mathrm{S} 1070427208020377$

The monograph by A.A. Popova summarizes and analyzes the results of numerous studies of the anodic behavior of a large group of metals, mostly in aqueous-organic and organic media. Special attention in the presentation of the material is given to the influence of complexation in solutions on the anodic behavior of metals and formation of anodic films. The book comprises an introduction, four chapters, conclusion, and a bibliographic list for the monograph as a whole.

The brief introduction (pp. 5, 6) substantiates the topicality of the issues considered in the monograph and notes the importance of the influence exerted by the type and properties of an electrolyte on anodic processes and on the formation and nature of the films formed. The first chapter (pp. 7-34) considers the fundamental aspects of the anodic behavior of metals passivated in aqueous-organic and organic media. The thermodynamic characteristics of the occurring processes, role of water in anodic reactions, and effect of anions on the anodic behavior of transition metals are discussed.

The second chapter (pp. 35-69) is devoted to the effect of complexation in solutions on the processes occurring in anodic polarization of metals. A section of the chapter contains evidence about application of chromatographic methods to determine transition metal ions in organic and mixed media. The third chapter (pp. 70-121) discusses a whole group of issues related to the nature of processes that occur on the surface of passivable metals, structure of the double layer, and formation of the metal-electrolyte interface.

The fourth chapter (pp. 122-154) includes material about the passive state of transition metals and the structure of oxide films on these metals. Also, some data obtained in studies of the semiconducting properties of anodic films are presented. In the conclusion (p. 155), the authors express hope that the monograph offered to readers' attention and based on a vast body of processed information sources will make up for the deficiency of review information about the problem discussed. The bibliographic list (pp. 156-183) is, indeed, rather spacious, with 503 references included. Unfortunately, the article titles are not presented, which somewhat reduces the information value of the list.

There is no escape from mentioning the shortcomings of the book, which are, in our opinion, due to two main reasons, lack of author's standpoint concerning the problems discussed and attempt to cover a too wide variety of issues. In fact, the monograph is a review of published information within rather uncertain limits. There are no sufficient interrelations between the chapters and between sections within the chapters. Separate publications are taken and an attempt is made to present their essence in an as brief manner as possible. What is the relationship between the phenomena of anodic dissolution, passivity, and entropy of the double layer. This is difficult to understand from the text in pp. 103-109.

There are numerous minor defects of presentation, which hinder the understanding of the material, both in the text and in the figure design. There is no such notion as "activity coefficient of the medium" (p. 7). The term "free energy" was many years ago replaced in the world literature with the term "Gibbs energy." What is the terminological difference between the heat $(\Delta H)$ of the sorption process (Figs. 2.4, 2.5) and heat of adsorption $(Q)$ (Figs. 2.6, 2.7). In Table 2.2, the symbol $\Delta H$ already designates the "differential molar energy of sorption." The least attention is given by the book to electrochemical processes: anodic dissolution and transition to the passive state. Anodic polarization curves are shown only in a single figure (Fig. 1.3).

The author should have taken as an example of a book devoted to a similar problem the monograph by B.N. Kabakov Elektrokhimiya metallov $i$ adsorbtsiya (Electrochemistry of Metals and Adsorption, Moscow: Nauka, 1966, 222 pp.). Published 40 years ago, this book still retains its scientific value.

\section{A.G. Morachevskii and M.S. Kokhatskaya}

